Revue d'histoire de l'Amérique française

REVUE D.HISTOIRE DE L'AMÉRIQUE FRANÇAISE

\title{
Morale catholique et détresse conjugale au Québec. La réponse du service de régulation des naissances Seréna,1955-1970
}

\section{Diane Gervais}

Volume 55, numéro 2, automne 2001

URI : https://id.erudit.org/iderudit/010361ar

DOI : https://doi.org/10.7202/010361ar

Aller au sommaire du numéro

Éditeur(s)

Institut d'histoire de l'Amérique française

ISSN

0035-2357 (imprimé)

1492-1383 (numérique)

Découvrir la revue

Citer cet article

Gervais, D. (2001). Morale catholique et détresse conjugale au Québec. La réponse du service de régulation des naissances Seréna,1955-1970. Revue d'histoire de l'Amérique française, 55(2), 185-215.

https://doi.org/10.7202/010361ar
Résumé de l'article

Cet article repose sur les archives de Seréna, le premier service de régulation des naissances au Québec, et les témoignages oraux de ses fondateurs. Il étudie le travail d'éducation populaire de Seréna en s'attachant particulièrement au contexte culturel de son émergence et à la philosophie de l'amour qu'il véhiculait. Tout en propageant une méthode acceptée par l'Église, Seréna proposait une transformation des rapports hommes/femmes dans la sexualité. L'article analyse en deuxième partie un corpus de 145 lettres dans lesquelles des couples francophones catholiques exposaient leurs problèmes moraux, conjugaux et sexuels. 


\section{Morale catholique et détresse conjugale au Québec. La réponse du service de régulation des naissances Seréna, I955-1970}

DIANE GERVAIS

Département d'histoire

Université Concordia

RÉSUMÉ • Cet article repose sur les archives de Seréna, le premier service de régulation des naissances au Québec, et les témoignages oraux de ses fondateurs. II étudie le travail d'éducation populaire de Seréna en s'attachant particulièrement au contexte culturel de son émergence et à la philosophie de l'amour qu'il véhiculait. Tout en propageant une méthode acceptée par l'Église, Seréna proposait une transformation des rapports hommes/femmes dans la sexualité. L'article analyse en deuxième partie un corpus de I 45 lettres dans lesquelles des couples francophones catholiques exposaient leurs problèmes moraux, conjugaux et sexuels.

ABSTRACT - This article deals with moral issues raised by the problem of family planning among Catholics between 1955 and 1970. It studies the influence of Seréna, the first family planning service in Québec, with a special attention to the cultural context in which it developed, and to its philosophy. While advocating the adoption of a method for fecundity control accepted by Church, Seréna pleaded for a profound transformation of relation between men and women in sexual intercourse. Finally, this article analyses attitudes of francophone couples towards their moral, marital and sexual problems. This research is based on the archives of Seréna and oral testimonies of its founders.

1. Je collabore depuis plus de deux ans au projet de recherche de Danielle Gauvreau et Peter Gossage sur "Le déclin de la fécondité au Québec depuis la fin du $\mathrm{xIx}^{\mathrm{e}}$ siècle». La présente recherche a été subventionnée par la Fondation Hannah et par le Conseil de recherches en sciences humaines du Canada que je remercie sincèrement. Je remercie également Marie-Odile Magnan, de l'Université Concordia, assistante de recherche attachée au projet. Tout spécialement, je tiens à souligner la généreuse et chaleureuse collaboration que m’a apportée l'équipe Seréna actuelle. Sans la confiance qu'ils m’ont accordée, ce travail n'aurait pu être réalisé. 
D ANS UN ARTICLE consacré aux idéologies politiques au Québec, André J. Bélanger décrivait les années 1950 comme «une période morale avant d'être politique ${ }^{2}$ ». Ce qui suit est une sorte de variation sur ce thème. Si l'on regarde la question de la limitation des naissances telle qu'on l'abordait - ou ne l'abordait pas - à l'époque, on est frappé de voir qu'elle relevait exclusivement de la compétence du clergé. Il est vrai qu'elle posait une question morale capitale et qu’elle a entraîné «la crise morale de notre siècle», ainsi que le titrait le journal La Presse en $1969^{3}$.

Depuis l'encyclique Casti connubii, parue en 1930, la contraception était marquée dans l'ensemble des pays catholiques du sceau du malum in se : «l'acte du mariage est par sa nature même destiné à la génération des enfants », avait écrit Pie XI ; en conséquence, "ceux qui en l'accomplissant s'appliquent délibérément à lui enlever sa force et son efficacité agissent contre la nature ; ils font une chose honteuse et intrinsèquement déshonnête ${ }^{4} »$. La condamnation était sans équivoque. Suivant les pays toutefois, le clergé pouvait en atténuer la rigueur par une attitude pastorale prudente et discrète comme ce fut le cas notamment en France, comme l'a démontré brillamment Martine Sevegrand 5 . Dans le Québec de l'aprèsguerre, certains confesseurs très tournés vers Rome et attachés au concept de nature, bouleversaient par leur intransigeance la conscience des couples pratiquants. De son côté, l'Action catholique, soucieuse de l'harmonie des couples et de leur éducation spirituelle, diversifiait et intensifiait son action dans un impressionnant éventail de mouvements et associations voués aux questions familiales, ainsi que l'a démontré Marie-Paule Malouin dans une excellente synthèse consacrée aux mouvements familiaux ${ }^{6}$. Tout en préconisant une morale conjugale de "permis-défendu », ces divers mouvements s'employaient à la propagation d'une philosophie de l'amour comme don de soi et préconisaient la maîtrise sexuelle qui humanisait l'amour.

2. André-J. Bélanger, «Les idéologies politiques dans les années 1950 », dans J.-F. Léonard, dir., Georges-Émile Lapalme (Sillery, Presses de l'Université du Québec, 1988), 131.

3. Cardinal Karol Wojtyla, «Paul VI se prononce sur la crise morale de notre siècle» : dans un cahier reproduisant une série d'articles parus dans La Presse du 27 septembre au 7 octobre 1969, Droits réservés La Presse.

4. Les enseignements pontificaux, Le Mariage (Belgique, Desclée, 1956) : "Casti Connubii», art. $314: 208$.

5. Martine Sevegrand, Les enfants $d u$ bon Dieu. Les catholiques français et la procréation au $X X^{e}$ siècle (Paris, Albin Michel, 1995).

6. Marie-Paule Malouin, Le mouvement familial au Québec. Les débuts : 1937-1965 (Montréal, Boréal, 1998). 
Mais si les années 1950 ont été morales, la décennie suivante fut sociale, à la suite de la multiplication des acteurs sociaux concernés par le débat soulevé par l'épineuse question. À partir de 1960, d'autres voix s'ajoutèrent, changeant sensiblement la nature du discours. La question mobilisa d'abord timidement, puis avec éclat, une nouvelle génération d'intellectuels, de travailleurs sociaux et de médecins de toutes obédiences, et elle bénéficia d'une couverture médiatique d'une rare intensité ${ }^{7}$.

Pendant ces années-là, l'angoisse du clergé devint palpable. Le dilemme des prêtres était de demeurer fidèle à l'Église tout en apportant un soulagement aux couples dont la détresse paraissait sans issue. Avec le Concile Vatican II (1962-1965), un espoir naquit et la fièvre pastorale culmina ${ }^{8}$. L'Église était très divisée. Pendant cette période de recherche, une partie du clergé se mettait à douter et adoptait une attitude de réserve discrète, si bien qu'un changement irréversible du discours et des pratiques s'amorçait. Au Québec, plusieurs sonnaient l'alarme ou s'engageaient ouvertement, notamment les dominicains Marcel-Marie Desmarais, Georges Méthot et les séculiers Guy Brouillet, Georges Matte et Jules Parenteau, pour ne nommer que ceux-là'.

En 1964, des laïcs convaincus s'émouvaient de ce qu'ils appelaient la «loi du minimum moral» chez les «couples moyens» ainsi que du climat d'anomie qui régnait dans notre monde pluraliste. Ils déploraient également le manque "de consensus positif sur la morale catholique ${ }^{10}$ ». Si la morale avait déjà été claire, il faut reconnaître que, dans les années 1960, il régnait en ce domaine plus de confusion que de clarté.

Pendant les années conciliaires, la pilule commençait à se répandre. En outre, la régulation des naissances émergeait comme un problème social en raison de l'intérêt que les travailleurs sociaux portaient aux populations défavorisées. La surpopulation du globe, à la une des journaux, contribua également pour beaucoup à cette intrusion du problème dans

7. En particulier les démographes Hubert Charbonneau et Jacques Henripin, la sociologue Colette Carisse, la journaliste Alice Parizeau, les docteurs Serge Mongeau, Maurice Jobin et Lise Fortier, figures de proue de la campagne en faveur de la contraception. Voir «Alice Parizeau ouvre le dossier du contrôle des naissances", Châtelaine (juin 1963) : 24 et ss., et «L’enfant a-t-il le droit de naître? La régulation des naissances est le problème de l'heure ", La Patrie, 26 novembre 1964.

8. Georges Méthot, "Beaucoup de méthodes peu de solutions», Maintenant, 24 décembre 1963, 365 ; Martine Sevegrand, op. cit., 234.

9. Marcel-Marie Desmarais, op, "L’Église ne rejette pas la régulation des naissances", $L a$ Presse, 30 octobre 1963; Georges Méthot, op. cit.; Guy Brouillet, prêtre, Sexualité et vie chrétienne (s.é., s.d.).

10. Fonds Seréna-Québec, Programme d'une réunion de l'Équipe de Saint-Jérôme, 4 mars 1964. 
les affaires publiques. On commençait à parler du droit à une contraception efficace.

En dépit de cette puissante vague de fond qui touchait aussi bien les laïcs que les clercs, à la stupéfaction générale, en juillet 1968, le pape trancha en appuyant l'aile conservatrice minoritaire de la commission qu'il avait lui-même créée, mettant en échec la voix majoritaire ${ }^{11}$. L'encyclique Humanae vitce réitérait la position exprimée par Casti connubii. Elle reprenait l'interdit moral, mais elle donnait une note d'espoir pour les couples dans les principes d'une "morale de cheminement» où les fautes étaient pardonnées dans la mesure où le pécheur tendait sincèrement vers la perfection. L’ancienne morale du permis-défendu était chose du passé. Mais le monde catholique n'a entendu que le vacarme assourdissant du NON! L'Église romaine paya sa rigueur de la défection de très nombreux fidèles.

À Montréal, en 1955, alors que tout ce qui touchait de près ou de loin la sexualité demeurait tabou, un service de régulation des naissances était mis sur pied par des laïcs. Plus tard, il prit le nom de Seréna (SErvice de RÉgulation des NAissances) qui évoquait en même temps la sérénité apportée aux couples. Il fut le premier à s'afficher publiquement, à proposer ouvertement une solution, alors que l'interdit touchant la publicité des moyens contraceptifs figurait toujours au Code criminel canadien. En dépit de périodes de doute, ce service laïque demeura fidèle à la morale catholique. S'il consacrait l'essentiel de son énergie à l'enseignement d'une méthode de continence périodique, il renseignait néanmoins sur l'ensemble des procédés contraceptifs disponibles dans le respect de la différence, une valeur de plus en plus affirmée dans les années 1960.

Dans cet article, je présenterai d’abord Seréna; peu étudié jusqu’à ce jour, si ce n'est dans les pages que lui consacre Marie-Paule Malouin dans l'étude déjà citée ${ }^{12}$. Je rappellerai brièvement l'histoire des débuts de l'organisme, alors qu'il occupait quasiment seul le champ de la planification familiale au Québec, soit la période entre sa création en 1955 et la publi-

11. Pour l'histoire de la commission pontificale, lire, entre autres, Robert McClory, Rome et la contraception. Histoire secrète de l'encyclique Humanae Vitæ (Paris, Les éditions de l'Atelier, 1998, première édition 1995). Je dispose également d'un entretien que m’a gracieusement accordé un médecin membre de la commission pontificale.

12. M.-P. Malouin, op. cit., 114-118; Diane Gervais, «Seréna et l'éducation populaire à la régulation des naissances, 1955-1968", Supplément au SEN, 24,4 (automne 2000) : 1-15. Voir également Jean-Marc Larouche, Éros et Thanatos. Sous l'œil des nouveaux clercs : essai socio-historique sur la sexologie et la thanatologie dans la société québécoise (Montréal, VLB, 1991), 40-43; le Collectif Clio, L'histoire des femmes au Québec depuis quatre siècles (Montréal, Quinze, 1982), 401-402. 
cation de l'encyclique Humanae vitce en 1968. J'évoquerai également sa philosophie de l'amour et du mariage, qui remettait en question la division des rôles dans la sexualité et assainissait le climat des décisions du couple en matière de planification familiale. Dans un second temps, j'exposerai la détresse sexuelle et morale des couples canadiens-français à partir de la correspondance reçue par l'organisme. Dans une perspective d'étude des représentations dans le domaine de la morale individuelle et sociale, je voudrais mettre en lumière les problèmes moraux et conjugaux vécus par une partie de la société québécoise catholique et montrer que le travail d'éducation populaire de Seréna constitua un jalon de la mutation culturelle du tournant de la Révolution tranquille. Contrairement aux idées reçues, est mis de l'avant l'apport de la philosophie catholique de l'amour aux récentes transformations des rôles masculins et féminins dans le couple. En même temps sort de l'ombre une population qui avait intériorisé et tenté de mettre en pratique l'exigeante morale conjugale catholique au prix d'une extraordinaire tension psychologique et morale.

Mes sources sont essentiellement les témoignages de membres de Seréna et les archives du groupe. Des entretiens en profondeur ont été réalisés avec Rita Breault, Yolande Provencher et Suzanne ParenteauCarreau, m.d. Les archives de Seréna sont très riches : ont été consultés des procès-verbaux de réunions, des textes de morale, des manuels de formation des moniteurs et la correspondance des couples consultants ${ }^{13}$. Ces sources disent beaucoup sur les préoccupations de l'époque et sur la culture des militants de l'action catholique et des familles qui avaient recours aux services de Seréna. L'étude a pour but de comprendre de l'intérieur un système de représentations partagées. Pour ouvrir la perspective à l'aspect dynamique des représentations sociales, j'aurai recours accessoirement à des sources complémentaires, notamment les journaux de l'époque, les archives d'associations de planification familiale nées dans les années 1960, ainsi que les témoignages de 16 prêtres et d'une vingtaine de médecins que j’ai recueillis pour la recherche sur «le déclin de la

13. Le fonds d'archives de Seréna-Québec n'étant pas encore catalogué, il m'est impossible de fournir les cotes des pièces citées. Le dépôt de ces documents à la Division des archives de l'Université de Montréal est actuellement en cours. J'ai eu le privilège de les consulter avant qu'ils soient rendus publics et j'ai contribué à leur mise en ordre et à la rédaction du contrat de donation. Tout au long de ce processus, la fondatrice, Rita Breault, s'est montrée soucieuse de ne pas trahir l'histoire. Il m'a semblé qu'elle retenait tout ce qui relevait du domaine public sans égard à l'image du groupe qui pouvait être projetée, n'éliminant que ce qui avait un caractère privé. 
fécondité au Québec» lancée par Danielle Gauvreau et Peter Gossage, à laquelle je collabore.

L'influence de l'Église sur les comportements des couples en matière de sexualité et de contrôle des naissances n'est pas une question nouvelle dans l'historiographie québécoise. Tout récemment, dans cette même revue, le sujet a fait l'objet d'un article de Gérard Bouchard auquel je renvoie le lecteur intéressé à une revue de littérature conséquente. L'intérêt de l'article de Bouchard réside en ce qu'il met en évidence la volonté de résistance aux interdits religieux des couples de l'ancienne paysannerie saguenayenne et, surtout, la possibilité d'une réelle volonté contraceptive en dépit d'une fécondité élevée indiscutable résultant de l'utilisation de moyens inefficaces. Pour sa part, Denyse Baillargeon, dans son livre Ménagères au temps de la crise, avait observé le même phénomène à partir de trente témoignages de Montréalaises. L'auteure montre que la plupart d'entre elles avaient utilisé des moyens contraceptifs défendus, mais elle fait remarquer en même temps l'intériorisation de la norme religieuse, nettement visible dans la culpabilité entraînée par cette transgression ${ }^{14}$.

Enfin, dans l'article «Empêcher la famille : fécondité et contraception au Québec, 1920-1960", en s'appuyant sur les recensements, sur l'enquête de fécondité du Québec de Jacques Henripin et sur les témoignages recueillis par Denyse Baillargeon, Danielle Gauvreau et Peter Gossage observent un lent déclin de la fécondité au Québec à partir de 1870; déclin qui laisse supposer des pratiques de régulation, lesquelles seraient différentes selon que l'on est catholique ou non. Après les années 1930, suivant les auteurs, 80 pour cent des catholiques auraient eu recours à la méthode Ogino, qui n'entrait pas en contradiction avec leur foi, contre 15 pour cent seulement chez les non-catholiques. L'influence de la religion sur les pratiques contraceptives paraît donc incontestable ${ }^{15}$. Le présent article s'inscrit dans la foulée de ces études mais, en raison du caractère particulier des sources, qui expriment un point de vue unique, celui d'un milieu catholique militant, il sera moins question de la transgression des normes que de leur intériorisation.

14. Gérard Bouchard, «La sexualité comme pratique et rapport social chez les couples paysans du Saguenay (1860-1930)", Revue d'histoire de l'Amérique française, 54,2 (automne 2000) : 183217, et Denyse Baillargeon, Ménagères au temps de la crise (Montréal, Les Éditions du remueménage, 1993), 99-110.

15. Danielle Gauvreau et Peter Gossage, «Empêcher la famille. Fécondité et contraception au Québec, 1920-1960», Canadian Historical Review, 78,3 (septembre 1997) : 478-510. 


\section{SERÉNA}

\section{Historique}

Issu, en 1955, de la Ligue ouvrière catholique (LOC), Seréna fut très vite pris en charge par les laïcs qui en avaient été les promoteurs, contrairement à la tradition lociste qui voulait que ses dirigeants laïques travaillent sous la dépendance étroite de la hiérarchie cléricale ${ }^{16}$. Comme l'a écrit Marie-Paule Malouin, ce groupe naissait à la suite de nombreux mouvements consacrés à la spiritualité des couples, mais il se donnait une mission beaucoup plus concrète ${ }^{17}$.

C'est Georges Matte, vicaire de la paroisse Sainte-Françoise de Lachine, qui avait demandé à Gilles et à Rita Breault d'assurer l'une des séances du Service d'orientation des foyers (SOF) de la paroisse, dans le but d'y exposer la nouvelle méthode de régulation des naissances qu'ils avaient euxmêmes expérimentée. Comme on sait, l'Église n'approuvait alors que la continence absolue ou périodique, incarnée dans la méthode Ogino ou "du calendrier», connue depuis le début des années 1930, et qui n’est valable que pour les femmes au cycle régulier. On sait par ailleurs que la pratique du coït interrompu bénéficiait d'une tolérance inconstante et capricieuse. Les couples catholiques vivaient à l'époque une crise aiguë. La méthode sympto-thermique (dite aussi du thermomètre) que ce jeune couple proposait commençait à être diffusée dans certains pays d'Europe, mais elle était inconnue au Québec. Son intérêt résidait dans ce qu’elle garantissait une grande sécurité aux couples, pourvu qu'ils ne prennent aucun risque, qu'ils l'utilisent correctement (ce qui n'allait pas de soi) et, surtout, de manière stricte, c'est-à-dire en ne s'autorisant des relations complètes que dans la phase post-ovulatoire qui dure de dix à douze jours par cycle $^{18}$. En revanche, elle pouvait exiger des couples dont la femme était irrégulière "des carêmes » assez longs puisque seuls les quelque douze der-

16. Gabriel Clément, Histoire de l'Action catholique au Canada français (Montréal, Fides, 1972), 15 et 145 .

17. M.-P. Malouin, op. cit., 114.

18. Docteur S. Parenteau-Carreau, "Seréna, ses lignes de force», Le médecin du Québec, 8,8 (août 1973) : 23. Docteur C. Tietze classe la méthode (utilisée de manière stricte) parmi les méthodes de contraception les plus efficaces, après les anovulants et avant le stérilet : "Ranking Contraceptive Methods by Level of Effectiveness, Advances in Planned Parenthood", Proceedings, VIII Annual Conference American Association of Planned Parenthood Physicians (Boston, avril 1971), vol. 6. Amsterdam/New York, Exerpta Medica, 1971, 117-126. Toutes les études d'efficacité comparée des méthodes contraceptives signalent l'importance de distinguer entre efficacité théorique et efficacité pratique, de même que l’importance de la motivation. 
niers jours de chaque cycle étaient absolument sécuritaires. Suzanne Parenteau, qui rejoignit très tôt la jeune équipe pour se consacrer en particulier à la recherche sur l'efficacité de la méthode, la décrivait ainsi :

Pour des centaines de couples qui, de toute façon, faisaient la continence périodique, cette méthode présentait une amélioration notable en présentant une base scientifique plus solide : la constatation de l'ovulation du cycle en cours plutôt qu'une probabilité mathématique de la période fertile. Pour ceux qui se fiaient uniquement à la relation interrompue, la méthode symptothermique signalait à chaque cycle cette période infertile d'une dizaine de jours où le couple pouvait avoir des relations complètes et indiquait cette phase ovulatoire où la relation interrompue présentait le plus de risque ${ }^{19}$.

À la fin de l'année 1955 donc, le couple Breault enseignait la nouvelle méthode aux couples du Service d'orientation des foyers de sa paroisse. Rapidement saisis par l'urgence des besoins et à la suggestion de leur

F I G U R E I

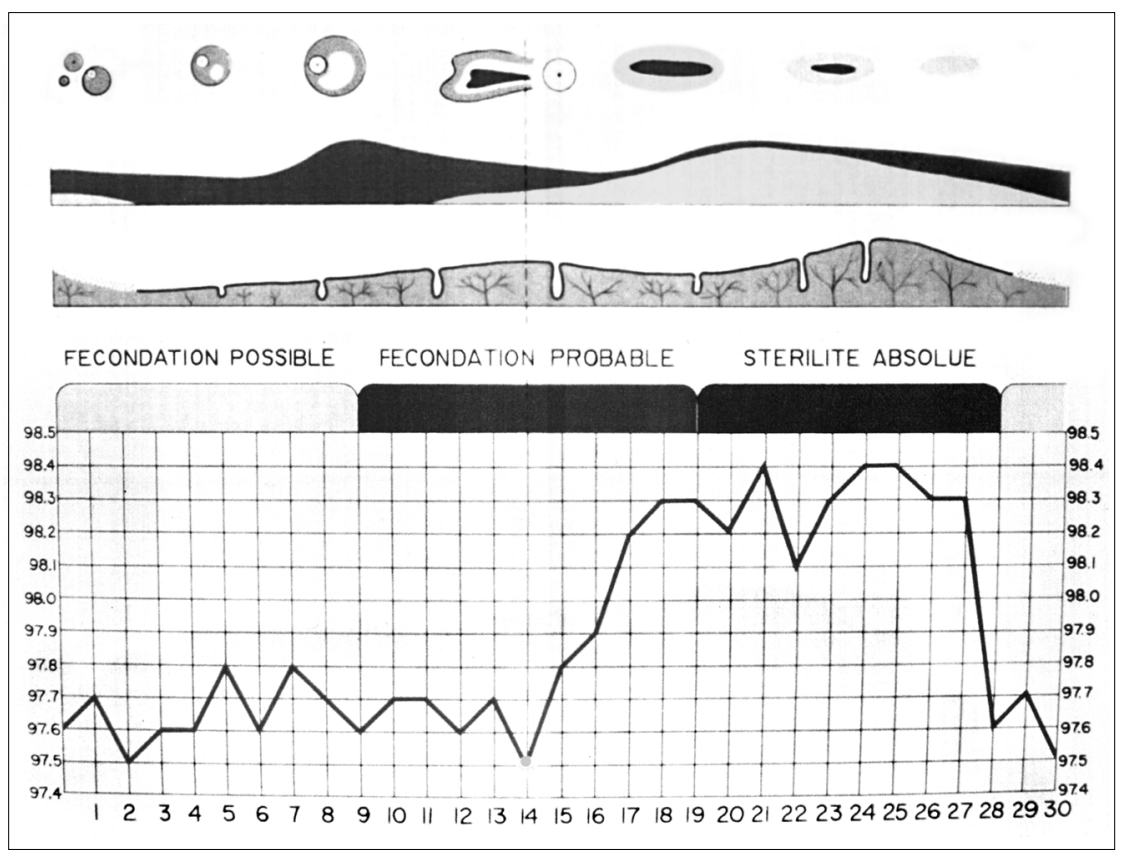

Planche d'enseignement illustrant les 3 phases du cycle ovulatoire: fécondation possible, fécondation probable, stérilité absolue (Archives Seréna-Québec)

19. S. Parenteau-Carreau, op. cit., 22. 
F I G U R E 2

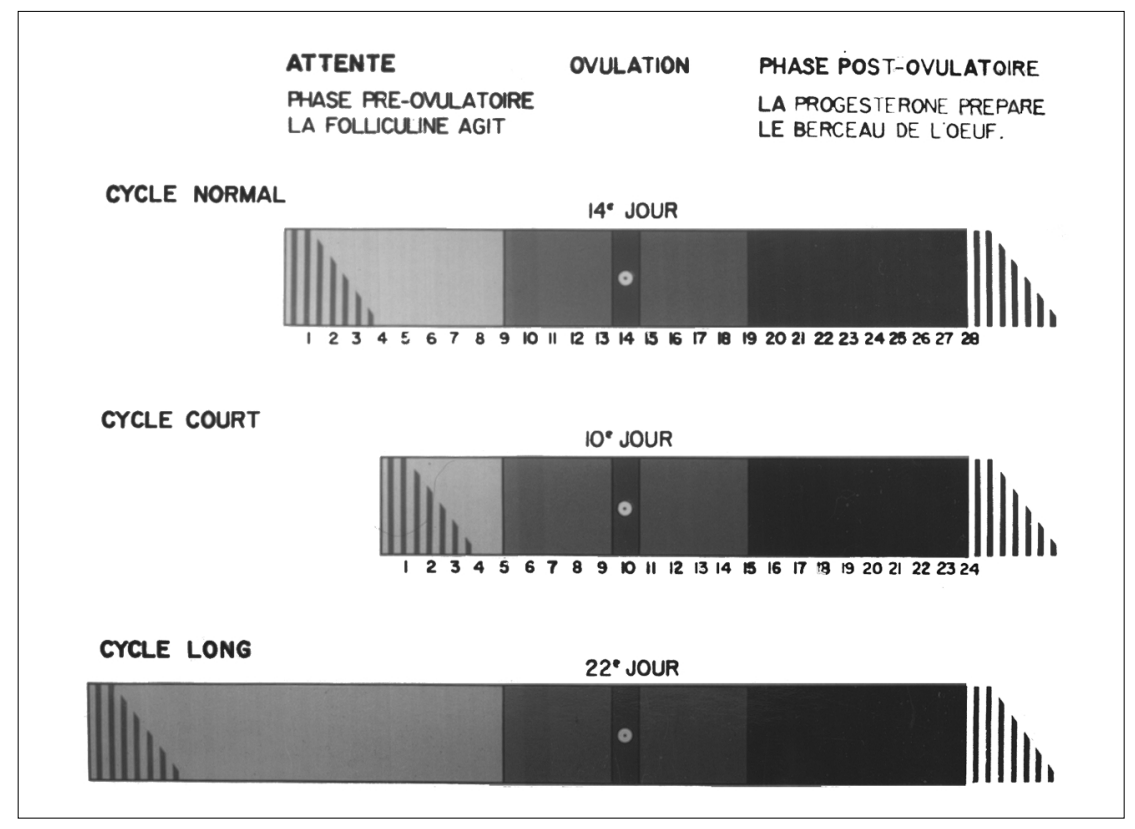

Planche d'enseignement (Archives Seréna-Québec)

vicaire, l'abbé Georges Matte, ils se mirent parallèlement à recevoir des couples à leur domicile. On allait chez eux comme chez le guérisseur : avec angoisse, après avoir tout essayé et en se disant qu'on n'avait rien à perdre. Parfois, des couples qui avaient pris rendez-vous s'arrêtaient brusquement devant la porte pour s'en retourner sans avoir osé frapper. Parler de ces choses-là n'était pas facile, tout comme entendre les mots qui nommaient "par leurs vrais noms» les choses du sexe.

Après la première séance d'enseignement, Rita Breault et par la suite les monitrices qui se sont jointes à l'équipe assuraient un suivi journalier pendant un, deux ou trois mois. Il s'agissait pour elles de conserver un graphique pour chaque femme suivie sur lequel elles inscrivaient le degré de température vaginale communiqué par téléphone. L’ovulation confirmée, il restait à rassurer les couples sur la sécurité de leurs relations conjugales. Ce suivi assidu était indispensable pour soutenir les couples dans le difficile apprentissage de la méthode.

La force de leur enseignement reposait sur l'approche qu'ils appelaient «de couple à couple», qui veut que l'enseignement soit donné par un 
couple à un autre couple. Cette technique tablait sur l'exemple d'un couple ordinaire qui offrait le témoignage vivant et chaleureux que la continence amoureuse était possible et épanouissante ${ }^{20}$. Une lettre adressée à Rita Breault par un petit employé montréalais témoigne du lien particulier qui se créait entre les couples consultants et leurs moniteurs lorsque le courant passait :

Au cours de notre entretien, j'ai remarqué qu'il existait entre vous et votre mari un petit quelque chose que je ne peux définir mais que nous ne possédions pas. Mais ce petit quelque chose, je crois l'avoir trouvé depuis que nous suivons cette méthode du thermomètre. Ma femme est complètement changée. D’abord elle n'est plus nerveuse et n'est plus contractée au moment de nos rapports conjugaux. Indifférente qu'elle était à mes caresses, elle devient plus participante, en un mot elle est plus amoureuse, plus charmante... (lettre 4)

Des couples convaincus des avantages de cette méthode qui, plus qu'un moyen de régulation des naissances, devenait un mode de vie et une manière de vivre sa sexualité, firent le saut et se joignirent à l'équipe Seréna en devenant moniteurs. C'était le mode essentiel de recrutement. Une lettre adressée à Rita Breault par une femme qui souhaitait s'engager avec son mari à leur côté témoigne de l'état d'esprit de ces couples :

C'est avec un esprit d'apostolat et de charité chrétienne que nous voulons transmettre aux autres cette connaissance suffisante de la méthode symptothermique et de la maîtrise de soi-même. Sans la pratique de la méthode sympto-thermique et la sérénité dans laquelle nous vivons depuis deux ans je me demande si j'aurais désiré aussi ardemment une nouvelle grossesse. Tout le bonheur que nous goûtons grâce à votre travail persévérant, nous voulons, avec de nouvelles connaissances, le transmettre un jour à d'autres couples dans le besoin. [...] Là seules sont les raisons qui nous ont poussés mon mari et moi vers l'équipe Seréna. (Lettre 32)

Là résidait également la motivation du couple fondateur : résoudre le problème de la régulation des naissances, tout en transmettant les valeurs chrétiennes de maîtrise de soi. N’est-ce pas ce que l’Église tentait désespérément d'inculquer aux couples? Seréna ajoutait que cette maîtrise n'allait pas sans une connaissance précise et intime du cycle ovulatoire de chaque femme et, surtout, sans la sécurité qu'elle apporte.

20. Rita Breault, cinquième entretien, 4 mai 2000; S. Parenteau-Carreau, «The Couple-toCouple Approach in NFP», Bulletin Catholic Marriage Advisory Council, 124,31 (octobre 1991) : 16-19. 
Entre 1955 et 1962, la petite équipe des Breault travailla dans la plus grande discrétion, sans publicité aucune, à la suite d'une consigne du cardinal Paul-Émile Léger qui ne souhaitait pas voir des laïcs s'exprimer publiquement sur un tel sujet. Mais à partir de 1962, tout changea très vite. D’abord, il y eut un reportage de la Revue Populaire sur la méthode sympto-thermique et «le travail extraordinaire d'un couple qui préférait garder l'anonymat», puis, quelques mois plus tard, leur participation à l'émission télévisée de Françoise Faucher "Votre enfant Madame ${ }^{21}$ ». Ces événements assurèrent une plus grande visibilité. Les Breault furent dès lors beaucoup sollicités par les médias, ce qui leur valut plus d'un millier de lettres de couples en détresse.

À partir de cette année-là et pendant douze ans, grâce à Jules Parenteau, aumônier de l'hôpital Notre-Dame, ils offrirent chaque mois, dans l'amphithéâtre de cet hôpital, un enseignement public devant des auditoires qui comprenaient jusqu’à 150 couples les premières années, mais qui déclinèrent lorsque la pilule fut plus largement prescrite. Le succès de ce mode d'enseignement fut cependant mitigé, en particulier pour les gens de condition modeste. Plus de gens, certes, se trouvaient renseignés sur la méthode, mais encore fallait-il bien en assimiler toutes les finesses, ce qui était moins bien assuré dans ce climat où manquaient la chaleur et la force de conviction de la relation interpersonnelle.

Finalement, l'année 1963 fut marquée par la parution du livre La régulation des naissances. Précis de la méthode sympto-thermique du docteur Jacques Baillargeon et de la journaliste Hélène Pelletier-Baillargeon ${ }^{22}$. Ce jeune couple catholique, fasciné par le travail des Breault, s'était proposé pour réaliser avec eux ce livre qui fournirait un guide sûr aux moniteurs aussi bien qu'aux couples consultants. Mais les Breault, respectueux encore de la consigne du cardinal et souhaitant garder les coudées franches, n'y apposèrent pas leur signature; ils se contentèrent de figurer en dédicace. En 1969, 65000 exemplaires de ce livre furent vendus! Ce fut, dit-on, l'un des plus grands succès de librairie de l'époque après les célèbres Insolences $d u$ frère Untel. Écrit dans une langue précise, concrète, sur un ton léger, ses auteurs montraient que la méthode était relativement facile d'application

21. Renée P.-Rowan, «Une solution : la méthode sympto-thermique», La Revue Populaire (septembre 1962) : 35-37; «Votre enfant Madame», CBF-TV, Canal 2, 12 février 1963, Entrevue de Rita et Gilles Breault par Françoise Faucher, transcription intégrale, Fonds Seréna-Québec.

22. Jacques Baillargeon et Hélène Pelletier-Baillargeon, La régulation des naissances. Précis de la méthode sympto-thermique (Montréal, Les éditions du Jour, 1963). 
et sûre, quand elle était utilisée correctement. Et, surtout, toute la philosophie de l'amour enseignée par Seréna s'y trouvait exposée de façon claire et très convaincante pour les couples réceptifs aux exigences d'une vie conjugale chrétienne.

\section{La propagation d'une philosophie de l'amour}

Dans sa première charte, Seréna clamait l'esprit apostolique de son travail. Il voulait aider "tous les couples désireux de trouver des solutions humainement et spirituellement acceptables aux problèmes posés par la régulation des naissances", tout en adhérant "sans réserve à tous les principes de la morale chrétienne tels que professés et interprétés par l'enseignement officiel de l’Église». Dans la seconde charte, rédigée après la proclamation d'Humanae vitce (à laquelle Seréna s'est soumis), les termes "d'adhésion sans réserve» ne figuraient plus. Une légère distance était prise. Pour diverses raisons, Seréna tenait à son autonomie vis-à-vis du clergé $^{23}$. Celui-ci lui envoyait toutefois de la clientèle et servait de conseiller moral.

L'instrument didactique initial de Seréna était un diaporama audiovisue $^{24}$. Il comprenait des diapositives présentant tour à tour des représentations bibliques, des dessins des organes génitaux féminins et masculins, un thermomètre, les phases du cycle menstruel et des courbes graphiques. La bande sonore d'accompagnement faisait entendre un texte lu à trois voix qui représentaient le prêtre, le mari et l'épouse. Typique de son époque, cette leçon exprimait naïvement une sensibilité religieuse où l'amour humain, la procréation et la méthode sympto-thermique elle-même étaient perçus en continuité symbolique avec la Genèse, comme des prolongements du plan divin de la création du monde.

Le texte était divisé en quatre parties. D’abord, la voix de l’abbé Matte, citant la Genèse, rappelait que l'amour de l'homme et de la femme a été voulu par Dieu pour qu'ils puissent collaborer à la création du monde. Gilles Breault enchaînait sur les inquiétudes des fidèles devant l'obliga-

23. Fonds Seréna-Québec, Les Équipes Seréna, fins, moyens, modalités, membres (1963); Statuts et règlements, Seréna inc. (1972). Le Mémoire soumis à la Commission d'Étude sur les lä̈cs et l'Église (1970) montre l'ambiguïté du statut du groupe par rapport aux autres groupes de l'Action catholique. Il se définit comme n'étant pas confessionnel et n'ayant aucun lien structurel avec l'Église bien que la plupart de ses membres soient catholiques pratiquants.

24. Mylaine et Aimé Denis, le premier couple à avoir rejoint les Breault et qui fondera le SERF, avait collaboré à la réalisation du diaporama, ainsi que le signale M.-P. Malouin, op. cit., 115, 117. Fonds Seréna-Québec, Diaporama. 
tion de la procréation, inquiétudes auxquelles répondait l'abbé rappelant la sollicitude de l'Église, son acceptation d'une prudente régulation des naissances par la continence périodique. Finalement, Rita Breault exposait les aspects techniques de la méthode et les conseils pratiques utiles. Puis venait la conclusion heureuse de la paix de l'âme retrouvée.

En plus de la Genèse, l'abbé Matte s'était inspiré des derniers enseignements pontificaux sur la régulation des naissances dont il tirait habilement parti. Par là, il voulait rassurer les couples sur le caractère licite de la nouvelle méthode. Puis, en cédant la parole à Rita Breault pour l'enseignement de la méthode, il suivait la recommandation de Pie XII qui avait confié cette responsabilité à des femmes laïques dans sa célèbre allocution aux sages-femmes d'octobre $1951^{25}$. Bien qu'orientée vers une préoccupation profane, l'action de Seréna était imprégnée de la morale catholique. Mais, face à l'urgence des besoins et pour apaiser la misère sexuelle des couples, il fallait concentrer l'enseignement sur les techniques préconisées par la méthode et sur les aspects psycho-sexuels de la vie conjugale.

Par principe, Seréna n'acceptait jamais de donner un enseignement à la femme seule. Les couples moniteurs s'adressaient à des couples. Dans le but de donner une place significative au mari, ils affirmaient la nécessité de sa participation en toutes choses. On insistait sur sa prise en charge des enfants au petit matin pour assurer à la mère les trois heures de repos indispensables à l'exactitude de la prise de température. Il était capital qu'il ne soit plus «celui qui doit s'abstenir» et la femme «celle qui fait la police dans le lit». Seréna proposait la continence périodique comme une «continence vécue dans les bras l'un de l'autre invitant les couples à exploiter la valeur tendresse de l'amour, même pendant cette période où la valeur qu'on pourrait appeler orgastique est mise en veilleuse ${ }^{26} »$. Les couples, dont la vie sexuelle était perturbée par l'angoisse de la grossesse ou dont les relations sexuelles étaient devenues mécaniques, découvraient ainsi "toute une gamme de gestes inconnus ou oubliés » qui venaient rafraîchir le climat de leurs relations. Les périodes de continence prenaient, grâce à cet enseignement, un sens positif et thérapeutique. On avait coutume de les comparer aux fiançailles qu'on revivait chaque mois et qui entretenaient la flamme du désir contribuant à l'épanouissement de l'amour. 
Seréna comprenait que le salut des couples passait par une transformation des rôles, notamment dans les relations sexuelles ${ }^{27}$. Jean-Marc Larouche a soutenu, dans son livre Éros et Thanatos, que Seréna avait posé au Québec les bases d'une "sexologie catholique militante ${ }^{28}$ ». Ne pourrait-on pas avancer que, par le simple fait qu'elle exigeait en toutes choses la collaboration des deux époux, la méthode des températures telle que le groupe la proposait forçait un renouvellement des mœurs conjugales? Cette façon de voir allait à l'opposé de l'attitude féministe, laquelle préférait la prise en charge de leur fécondité par les femmes seules, ce que la pilule conférait. Toutefois, Seréna intéressera plus tard certaines féministes attirées par les méthodes naturelles de régulation des naissances.

\section{Diffusion de Seréna et caractérisation des couples consultants}

La popularité de Seréna grandit de manière fulgurante jusque vers le tournant des années 1970. Même à l'époque de son peu de visibilité, la méthode et sa philosophie emportaient la faveur d'un nombre important de couples comme l'attestent les statistiques compilées par la seconde présidente de Seréna, Marie-Paule Doyle, sur la base des fiches d'inscription des couples consultants ${ }^{29}$. Dans le tableau qui suit, les données agrégées pour les années comprises entre 1955 et 1964 recensent 7294 couples québécois ayant reçu au moins un enseignement. En 1964, une trentaine d'équipes se formaient à travers la province, si bien que l'année 1966 enregistrait le nombre record de 5107 couples avant que la régression commence. Sur l'ensemble de la période visée, 32346 couples, au total, avaient suivi au moins un enseignement. Si ces chiffres ne disent rien du nombre de personnes qui ont persévéré dans l'application de la méthode, ils montrent néanmoins les énormes besoins en matière de planification familiale répondant aux exigences de la morale chrétienne. Ce dont fait foi également l'immense succès de librairie du livre des Baillargeon.

27. D. Gauvreau, op. cit., et G. Bouchard, op. cit., entre autres, ont fait état de la violence conjugale dans les relations sexuelles.

28. Jean-Marc Larouche, Éros et Thanatos, op. cit., 40.

29. Fonds Seréna-Québec (Activités, août 1971) : «L'évolution de Seréna à la lumière des statistiques annuelles». Seréna préparait annuellement des relevés statistiques pour ses propres besoins à partir des fiches d'inscription des couples consultants. Les totaux enregistrés sont certainement au-dessous de la réalité car ce n'est que depuis 1966 que les enregistrements se sont faits de manière plus systématique. À partir de cette date, une carte d'inscription uniforme a été utilisée par la plupart des équipes, mais elle n'était pas obligatoire. Les résultats affichés sont tout de même une bonne indication. De 1955 à 1966, Seréna ne desservait que le Québec. À partir de l'année 1966 s'ajoutèrent les premières équipes formées au Nouveau-Brunswick et en Ontario. 
TABLEAU 1

Nombre de couples rencontrés par Seréna entre 1955 et 1970

\begin{tabular}{c|c}
\hline De 1955 à 1964 inclusivement & 7294 \\
1965 & 3814 \\
1966 & 5107 \\
1967 & 4618 \\
1968 & 4069 \\
1969 & 3734 \\
1970 & 3710 \\
\hline TOTAL & 32346 \\
\hline
\end{tabular}

Qui étaient les couples consultants? Les parents des baby-boomers, mariés entre la fin de la guerre et le début des années 1960. Une répartition des couples selon la profession du mari montre que Seréna attirait peu l'élite bourgeoise instruite. Sa clientèle était avant tout constituée des classes moyennes et surtout populaires. En effet, plus de $57 \%$ provenaient de milieux ouvriers de toutes catégories et, pour le reste, de la couche moyenne supérieure, comme l'atteste le tableau suivant.

TA B LEAU 2

Répartition des couples rencontrés en 1970 selon l'occupation du chef de famille

\begin{tabular}{l|c}
\hline \multicolumn{1}{c|}{ Occupation du chef de famille } & Nombre de couples \\
\hline professionnels & 61 \\
semi-professionnels & 115 \\
gérants et administrateurs & 32 \\
petits administrateurs & 74 \\
cols blancs & 170 \\
ouvriers & 834 \\
cultivateurs & 70 \\
étudiants & 79 \\
non indiqué & 17 \\
\hline TOTAL & 1452 \\
\hline
\end{tabular}




\section{Seréna et les autres}

À l'exception du SERF (Service éducatif de régulation au foyer), né en 1961 d'une scission à l'intérieur de l'organisme, Seréna sera le seul à dispenser un tel service jusqu'à la fin de l'année $1964^{30}$. En octobre 1964 naissait une association multiconfessionnelle et bilingue, l'Association pour la planification familiale de Montréal (AFPM), qui diffusait de l'information sur tous les moyens contraceptifs. Puis, en 1967, les francophones voulurent une association autonome et se regroupèrent autour du docteur Serge Mongeau, alors directeur de l'APFM, qui fonda le Centre de planification familiale de Montréal (CPFM) voué à la formation des intervenants en matière de planification familiale. En outre, cette année-là, les Services de santé de la Ville de Montréal ouvrirent trois cliniques de planification familiale dans les quartiers les plus défavorisés de la ville. Deux hôpitaux lancèrent également des cliniques ${ }^{31}$. De leur côté, les médecins, dans leur cabinet, commençaient à proposer aux femmes d'autres moyens contraceptifs qu'ils jugeaient plus efficaces que la méthode symptothermique. La plupart d'entre eux dénigraient cette méthode, disant qu'elle ne convenait qu'à une minorité bien pensante et éduquée, ce que dément le tableau 2.

Suivant les docteurs Parenteau-Carreau et Mongeau, ces années-là furent des années de concertation entre tous les milieux d'une société montréalaise de plus en plus composite. Les associations, comme les congrès sur la planification familiale, réunissaient les tenants de toutes les tendances. Tous s'entendaient sur l'urgence sociale d'une action en ce domaine. Ainsi, Seréna participa pendant plusieurs années aux activités du CPFM. Plus que le débat autour de la pilule, ce fut le dossier de la légalisation de l'avortement qui mit fin à cette collaboration ${ }^{32}$. De cette diversité des intervenants en planification familiale naquit une perception plus pragmatique de la contraception. On parlait moins de la légitimité de la

30. Pour l'historique du développement du SERF, voir M.-P. Malouin, op. cit., 117-118.

31. Il s'agit de l'hôpital Notre-Dame et de la Miséricorde. Fonds CPFM, "Ressources communautaires en planification familiale en date du 14-12-1967». Une partie des archives de l'APFM et du CPFM ont été consultées dans les locaux de Seréna, le docteur Parenteau-Carreau ayant été membre du comité conseil du CPFM de 1967 à 1969.

32. D’après trois témoins clés, dont deux extérieurs à Seréna. À partir de la réunion du 21 novembre 1968, les procès-verbaux du CPFM montrent la défection progressive de quelques-unes des agences sociales dirigées par des religieux lors de la soumission du CPFM au gouvernement de sa position face à l'avortement : Fonds CPFM des archives Seréna non comprises dans le fonds Seréna-Québec. M.-P. Malouin note qu’un débat fut engagé entre les tenants de la pilule et leurs "adversaires». Une étude approfondie des sources mériterait d'être faite sur la question. 
régulation des naissances et de la valeur de la continence que du droit fondamental de l'Homme à la contraception. Les moyens ne furent plus jugés suivant des critères moraux mais suivant ceux de réversibilité, innocuité, efficacité et acceptabilité par le couple. Au tournant des années 1970, Seréna fut accusé de vouloir transmettre des valeurs familiales influencées par le modèle chrétien. Les associations, à l'époque, faisaient valoir en effet qu'une information de qualité en matière de planification familiale se devait d'être objective ${ }^{33}$.

\section{LA DÉTRESSE DES COUPLES CATHOLIQUES}

Après ce bref historique, intéressons-nous au désarroi des couples, tel qu'exprimé dans un ensemble de lettres qui nous fait entrer dans leur intimité. Écoutons-les parler, jeunes et plus âgés, issus des diverses régions du Québec et tout spécialement de la région montréalaise, et qui furent intimement concernés par la proposition de Seréna. Nous avons dépouillé 245 lettres, écrites entre les années 1959 et 1968. Ces témoignages dispersés ne se prêtent pas à une analyse statistique, mais ils ont valeur d'exemplarité. Ils sont la parole d'une catégorie de femmes prêtes à de grands sacrifices pour demeurer au sein d'une Église dont elles perçoivent, sans le critiquer ouvertement, le manque de compassion et de compréhension vis-à-vis de leurs difficultés. La qualité de l'orthographe et de la syntaxe de chacune des lettres donne un indice du milieu social des correspondants et reflète les données statistiques présentées plus haut ${ }^{34}$. Jusqu'en octobre 1962, elles viennent de la grande région métropolitaine et, par la suite, de tous les coins de la province. (Voir en annexe la répartition des lettres citées.)

Les lettres se partagent grosso modo en trois groupes : les demandes d'aide souvent pressantes, les réponses à un appel de collaboration à une

33. Fonds d'archives du Centre de planning familial du Québec (CPFQ), Université du Québec à Montréal, Service des archives et de gestion des documents, fonds 113P, 203a/2, «Projet conjoint (entre l'APFM et le CPFQ) de centre d'information en planification familiale», octobre 1971.

34. Afin de préserver les indices de l'origine sociale des auteurs des lettres et leur force d'évocation, nous citerons les lettres en respectant les fautes d'orthographe et de syntaxe. Pour en faciliter la lecture toutefois, nous rétablirons le découpage morphologique de la langue standard, de même que nous compléterons au besoin la ponctuation. Par exemple, dans notre transcription, «ces» lorsqu'il veut dire "c'est» devient «c'es», "savoir» qui veut dire «s'être» devient "s'avoir», etc. Pour protéger l'anonymat des personnes, les noms précis des lieux d'où elles proviennent ainsi que tout autre renseignement permettant l'identification des personnes ont été omis. Un inventaire des lettres citées est fourni en annexe. Pour faciliter les renvois, chacune des citations sera suivie du numéro de la lettre entre parenthèses. 
étude d'efficacité de la méthode ${ }^{35}$ et les nombreux témoignages de reconnaissance, qu'ils soient spontanés ou au contraire sollicités par Rita Breault pour servir d'exemples de réussite aux femmes qui hésiteraient à utiliser la méthode ou se décourageraient devant la difficulté. Suivant la formule de l'apostolat «du semblable par le semblable», elles sont là pour dire : des gens comme vous l'ont fait; c'est pourquoi certaines d'entre elles peuvent paraître excessives ou trop élogieuses.

Une centaine d'entre elles sont techniques : examens de graphiques, conseils pratiques, etc. Nous les avons écartées, bien qu'elles fassent valoir la générosité, le sérieux et les difficultés énormes du travail de Seréna. Les 145 restantes sont au contraire très expressives, voire pathétiques, oscillant entre courage et désespoir, manifestant rarement de la révolte, plutôt de la soumission et de la bonne volonté. Elles disent la détresse sexuelle, conjugale, morale et sociale de leurs auteurs. L'accent de certaines d'entre elles laisse penser que Seréna a libéré un flot de paroles trop longtemps contenu : «fini les secrets d'alcôve» (lettre 34). Toutes représentent la parole de femmes (et d'hommes) catholiques qui, en dépit des difficultés de leur vie, ont cherché une solution morale à leur problème de régulation des naissances ${ }^{36}$.

\section{Des témoignages convergents sur fond de misère sociale et morale}

Le problème de la régulation des naissances se posait sur l'arrière-fond d'une grande misère sociale. La dépression nerveuse, la maladie, les problèmes de santé entraînés par les grossesses répétées, la pauvreté, le chômage, les maigres salaires, la faim ou le froid sont dépeints avec des accents parfois pathétiques :

Mon père, $j$ 'aimerais commencer ma lettre ainsi car je vous écris et je sais qui vous êtes. S'est un peu gênant pour vous : mon français est blessant car il est aussi pauvre que moi. Mais s'est en pensant à mon malheur à moi que je re-

35. Il s'agit de l'étude d'efficacité des docteurs Lanctôt et Parenteau à partir des milliers de graphiques fournis par les couples utilisateurs dès 1959. Claude A. Lanctôt, md, mph et S. Parenteau-Carreau, md, Études d'efficacité des méthodes de planification familiale basées sur le test thermique (Seréna inc., 1971).

36. Des centaines de lettres semblables ont été recueillies au Canada et aux États-Unis auprès de catholiques engagés par Pat et Patty Crowley pour être présentées à la Commission pontificale. Celles-là, recueillies au milieu des années 1960, dans un tout autre but, celui d'infléchir l'Église, critiquent la méthode Ogino et de la température qui créent «une obsession du sexe tout au long du mois", Robert McLory, Rome et la contraception, op. cit., 86-89. 
vois celui des autres. Cette lettre est pour ceux qui ont de la misère à signer les noms et doivent regarder sur le poteau le nom d'une rue avant de l'écrire. [...] Je suis donc en contact avec eux et inutile de se dire que sa n'existe pas. Combien de choses nous semble incroyable au temps que nous vivons. Ex. : des gens manquent de tout. Personne ne le crois. Moi j'ai fait cette triste expérience. J'ai connu se que s'est que manquer de nourriture, de chauffage et d'eau, oui d'eau, parmi du monde qui donnais des restes a leur chien, que nous aurions mangé de bon cœur. Notre vie de misère est si incroyable que si je l'écrirais mon père, vous croyriez que je veux attirer l'attention. J’ai passé par tout se qu'une femme peut vivre de misère noire. Depuis ma connaissance, je me rappelle avoir eu faim. (lettre 35)

Les lettres attestent qu'en dépit du renouveau économique de l'aprèsguerre, une partie de la population québécoise, en milieu rural comme à Montréal, vivait des crises financières endémiques ${ }^{37}$. Par ailleurs, sur le plan moral, c'était l’impasse. Les couples catholiques étaient « déchirés entre le désir physique de l'un vers l'autre, le désir de limiter les naissances et le désir de vivre chrétiennement ${ }^{38}$ ».

La lettre suivante résume assez bien le ton que l'on retrouve dans plusieurs lettres. Après avoir fait le long récit de ses misères et celles de sa famille, puis remercié Dieu de ne pas les avoir abandonnés en dépit de leurs «défauts», une femme de Montréal écrit :

Voici la suite de mon histoire. [...] Je n'espérais rien de cette rencontre (avec le couple moniteur), car j'avais eu les derniers enfants en suivant différentes méthodes et nous étions comme des chats qui craignent l'eau chaude. De voir des gens comme vous et moi père et mère et face au même problème me donna confiance et ce jour là tout changea. J'ai repris la pratique de ma religion et je n'aurai jamais assez de temps pour remercier Dieu, d'abord de m'avoir donné un mari comme le mien avec ses défauts et ses qualités, ensuite de permettre que les couples comme ces gens là [les moniteurs] soient de plus en plus nombreux, car les personnes les mieux intentionnées qui n’ont pas vécu ce drame ne comprendront jamais ce que père et mère ressentent vis-à-vis de ce problème. $[\ldots]$

37. Les conditions de vie des milieux défavorisés montréalais m'ont été souvent dépeintes en entretien par les médecins. Voir l'article du docteur Gisèle Phaneuf, médecin chef de district sanitaire au Service de santé de la ville de Montréal, «Santé et milieux défavorisés », Bulletin d'hygiène, 56,1 (Ville de Montréal, 1970), 11-17, statistiques à l'appui.

38. Rita Breault, Les idées nouvelles nous viennent de la base (Montréal, Seréna inc., 1975), 4. 
Une note gaie pour terminer : ceux qui craignent que cette période défendue n'a aucune valeur, apprendront vite qu'un met rare aiguise toujours plus l'appétit que celui qui revient sur la table à chaque repas régulièrement. (lettre 9)

Voilà une femme qui paraît relativement sereine; pourtant son passé était lourd. "Voilà la suite de mon histoire», écrit-elle, une histoire en deux temps, scandée par la rencontre de Seréna vers 1960. Dans la première partie de sa lettre (non publiée parce qu'elle contient trop d'informations confidentielles), elle se dit pauvre, sans instruction et ignorant tout de la vie et de la sexualité à son mariage, à 16 ans, vers la fin de la guerre. Elle décrit le mariage comme «une obligation de passer à l'Église pour dire oui et après, endurer son mari ». En 1960, cette femme avait onze enfants. À partir du sixième, elle ne les désirait plus, la pauvreté l'en empêchait, comme elle dit.

Cette lettre suggère de façon allusive ce que d'autres diront de manière plus explicite ou plus expressive : la pauvreté, la peur de la sexualité à la suite de grossesses répétées, l'échec des méthodes contraceptives et le problème conjugal qui s'ensuit, de même que le bonheur de retrouver la pratique religieuse. Elle montre également les qualités de l'approche de Seréna, valorisant la méthode de couple à couple, et, pour finir, vante les effets positifs de la continence périodique sur la vie sexuelle et la vie conjugale.

Dans l'ensemble des lettres, d'autres thèmes surgissent avec une récurrence inégale, notamment les problèmes de santé des femmes, omniprésents, la difficulté de se renseigner auprès des médecins sur les méthodes contraceptives, le scepticisme de ces derniers quant à l'efficacité de la méthode sympto-thermique et ses difficultés d'application. Le thème majeur qui les traverse est la grande satisfaction reliée au sentiment de pouvoir enfin contrôler sa fécondité dans la paix de l'âme et l'harmonie conjugale retrouvée ${ }^{39}$. Et parfois, ayant compris comment maîtriser sa fécondité, une mère de famille nombreuse, après bien des conceptions non désirées, dit retrouver «la joie de porter un enfant le cour léger» (lettre 35).

\section{Les échecs du contrôle des naissances}

«Si vous cherchez une méthode facile, ne m’écoutez pas!» disait Rita Breault. L'apprentissage de la méthode était ardu et son application très

39. Rappelons que certaines de ces lettres ont été sollicitées pour témoigner de la valeur de la méthode et convaincre d'autres couples. Quoi qu'il en soit, spontanées ou non, elles témoignent toutes de l'intériorisation du système de valeurs prôné par Seréna. Entre autres lettres, témoignent de leur grande satisfaction du service rendu les lettres du Fonds Seréna-Québec portant, dans la liste en annexe, les numéros 1, 3, 4, 6, 8, 13, 18, 33, 35, 44, etc. 
exigeante, notamment au chapitre de la continence. Pourquoi les couples payaient-ils ce prix? Pour retrouver la paix intérieure, soit, mais également faute de moyens efficaces de contrôle des naissances. Avant le milieu des années 1960, le sentiment général était qu’on n’avait aucun moyen de contrôler sa fécondité : «On n'avait rien, fallait faire un nœud!» me dit un médecin en entretien ${ }^{40}$. Le clergé lui-même reconnaissait que la détresse conjugale paraissait sans issue par suite de l'imperfection des méthodes permises ${ }^{41}$. Les échecs des «moyens du bord» utilisés, et en particulier de la méthode Ogino, sont maintes et maintes fois signalés dans les lettres. «À force d'essayer toutes sortes de choses, avec ses essais, la famille grandit», écrit à l'abbé Parenteau une femme qui a mis au monde 11 enfants, l'air de dire que les méthodes contraceptives ne sont rien d'autre que des moyens de faire des enfants (lettre 5). Dans le même ordre d'idée, une autre soutient qu'avec la méthode du calendrier les enfants lui viennent plus vite qu'avant (lettre 24), tandis qu'une troisième n'a guère plus de succès : 13 enfants en moins de seize ans de mariage : «Sa fait plusieurs années mon médecin veut que je me repose, mais j’ésait bien des méthodes et cela ne fait pas. Nous avons de la bonne volonté tous les deux moi et mon mari, mais après tous ces sacrifices, je suis toujours tombé en famille.» (lettre 17)

Comme l'écrivait fort justement Gérard Bouchard, la volonté et les pratiques contraceptives peuvent être une réalité, même si leurs effets ne sont, en aucune façon, mesurables ${ }^{42}$. Avec l'arrivée du stérilet et de la pilule, tout changea; mais ces moyens ne furent prescrits de façon courante qu'après le milieu des années 1960 et les réticences étaient encore énormes, aussi bien d'un point de vue médical que psychologique et moral. Dans quelques lettres se lit en effet la crainte de la pilule : «Est-il vrai qu'elle donne le cancer?" s'inquiète une femme, tandis qu'une autre constate que, depuis qu' elle la prend, elle est "comme un frigidaire» $(\text { lettres } 41 \text { et } 43)^{43}$.

40. Médecin $n^{\circ} 7$. Entre autres, citons une lettre en provenance d'un village rural d'une mère de six enfants : «Je suis très irrégulière. Vous comprenez que c'est difficile de suivre des méthodes. Nous disons que si Dieu veut qu'on ait X enfants, il n'y a rien à faire.»

41. En particulier les dominicains Marcel-Marie Desmarais et Georges Méthot, op. cit.

42. G. Bouchard, op. cit., 210.

43. Dans mon corpus, la première mention de la pilule est faite en 1962 (lettre 10). La première femme qui dit la prendre, le fait par intermittence dans le seul but de régulariser son cycle (lettre 20), tandis qu'elle a été prescrite à une autre pour quelques temps après son accouchement de façon à laisser se reposer son organisme (lettre 40). En 1965, un médecin de Montréal la conseille dans un but strictement contraceptif (lettre 38). C'est la première mention de ce genre. Une femme avoue utiliser le diaphragme (lettre 14). 
À l'exception de la pilule, assimilée à un médicament, et du test de fertilité permis par l'Église ${ }^{44}$, Ogino est la seule méthode que l'on se permet de nommer par son nom. Pour les autres, on écrit pudiquement «des essais», "toutes sortes de choses», des "précautions». Qu'y a-t-il derrière ces euphémismes? Il n'y a pas lieu d'en discuter ici, mais tout porte à croire que le coït interrompu était assez répandu. Il n'est mentionné qu’une seule fois, en des termes très explicites. Selon Denyse Baillargeon, c'était une pratique courante au temps de la crise. Elle se cache derrière les expressions : «faire attention» et "fumer dans le salon et cracher dans la cuisine ${ }^{45}$ ».

\section{La peur de la sexualité et la faillite du mariage}

«Je fais attention, ne t'inquiète pas!» disaient les maris : comment, dans ces conditions, ne pas vivre l'amour dans l'obsession de la maternité? La perspective de la grossesse, comme le notait également Gérard Bouchard pour le Saguenay, enlevait beaucoup d'attraits à la relation conjugale ${ }^{46}$. Une femme, qui se faisait la porte-parole des plus démunies, les décrivait inquiètes, nerveuses et peureuses de se soumettre à l'union complète «dans le lieu prévu par la nature». Pour beaucoup, l’amour était devenu un «cauchemar». Quelles étaient les maladies de l'amour? D’après madame Breault : la frigidité et l'éjaculation précoce.

Des craintes superstitieuses persistaient dans certains esprits qui reliaient le seul fait de faire l'amour aux divers problèmes gynécologiques des femmes, telle cette pauvre femme au seuil de la ménopause et aux organes fatigués qui demandait si «l'acte du mariage» trop souvent répété pouvait «causer de l'affection à la matrice». Le lien bien réel quant à lui, entre l'amour et la maladie mentale ou l'extrême tension nerveuse est maintes fois répété dans les lettres. L'auteure de la lettre qui vient d'être citée écrivait que sa mère était enfermée à Saint-Jean-de-Dieu depuis nombre d'années parce que «l'acte du mariage lui attaquait les nerfs ${ }^{47}$ ».

44. Le test de fertilité était un autre moyen de déceler l'ovulation pour pratiquer la continence périodique. Des femmes s'interrogent sur son efficacité : lettres 2, 22 et 37.

45. «Mon mari a toujours éjaculé en dehors du vagin depuis 16 ans à l'exception de mes grossesses qui ont toujours été désirées» (lettre 42). Denyse Baillargeon, op. cit., 105 et Danielle Gauvreau, op. cit., 493, signalent l'importance du coït interrompu comme pratique contraceptive.

46. G. Bouchard, op. cit., 208. L'obsession de la maternité et la peur de la sexualité apparaissent dans de nombreuses lettres, par exemple, lettres 15, 16, 19, 35, 42, etc.

47. Lettre $\mathrm{n}^{\circ} 28$, d'une femme rurale : «Sa fait je vais faire une dépression nerveuse vu mon mari est bien affectueux vers moi. Il veut continuer l'acte de mariage et moi je ne sens plus aucun travail sur les organes. Quand j'ai mes règles, je les ai trop abondantes et s'es un rouge pâle. Veuillez me dire si s'es dangereux pour causer de l'affection à la matrice si l'acte de mariage se produit souvent.» 
Vivre comme frère et sœur paraissait à certains la seule solution, en particulier aux couples dont la femme avait un cycle trop irrégulier ${ }^{48}$. Mais à quel prix? Déséquilibre, terreur et faillite du mariage. «Il va de soi que ce sacrifice nous a apporté déséquilibre et même terreur», confie un enseignant de Montréal qui a pratiqué avec héroïsme une continence absolue pendant plus de deux ans (lettre 15). La continence périodique non plus n'était pas facile à vivre :

Pour nous une grossesse, c'était presque une question de vie ou de mort. [...] Et parce qu'il ne fallait pas que ça arrive, nous suivions la méthode Ogino. À cause de mon irrégularité, c'étaient des carêmes exagérément longs, avec des appétits poussés à bout, des nerfs à vifs, des chicanes, la nervosité et malgré tout, l'inquiétude perpétuelle. Nous étions au bord de la séparation. (lettre 30)

Les hommes comme les femmes se décourageaient. "Mon mari est désemparé et tout ce qu'il voudrait me dire s'arrête dans sa gorge.» (lettre 10). À la longue, la surexcitation nerveuse, les griefs, les conflits s'installaient et les mariages se brisaient "doucement mais sûrement". "Ce problème la est terrible comme on ne pourra jamais se l'imaginé et il peut tout briser entre deux êtres qui s'aiment pourtant vraiment du fond du cœur" (lettre 35). De fait, plusieurs femmes avaient peur que leur mari aille voir ailleurs, une circonstance atténuante en confession d'ailleurs (lettres 22, 29). Et quand il n'y avait pas d'angoisse, c'est qu'il y avait résignation, comme ce témoignage l'indique :

C'était une vie de sacrifices, mais sans épanouissement. Soit dans nos manières ou nos réactions nous découvrions le renoncement pour ainsi dire obligatoire. À la naissance de ma fille qui est aujourd'hui mon bébé de quatre ans et la troisième de la famille, nous constations que pour quelques années, il faudrait absolument poser des freins. Ces freins très brusques ne régalaient pas la situation d'une femme qui n'avait jamais eu le bonheur de la moindre satisfaction avec son mari dans dix ans de ménage. Je me suis toujours montrée raisonnable et résignée [...] Mais, sans m’en rendre compte, je vivais continuellement le cœur gros et un peu aigrie. J'étais une personne très maigre (88 livres environ). [...] mon mari ne voulait pas entendre parler de méthode. (lettre 39)

En prônant un amour de type oblatif où les époux «se sacrifient l'un pour l'autre», pour utiliser les termes d'une femme consultante, Seréna débordait le cadre strict de l'enseignement d'un moyen de régulation des

48. Lettres 12, 15 et 29. Nul autre que le célèbre père Méthot condamnait la continence complète dangereuse pour l'harmonie conjugale, Maintenant, op. cit., 365. 
naissances. Beaucoup de femmes vantaient les bénéfices de cette philosophie de l'amour sur l'attitude de leur mari. L'une d'entre elles remarquait en particulier sa capacité nouvelle à dire sa tendresse, lui qui n'avait pas auparavant les mots pour le dire :

Nous y [dans les rencontres Seréna] avons appris bien des choses. Nous nous étudions tous les deux, nous nous aidons l'un l'autre. Nous avons repris la mâ̂trise de nous-mêmes. Nous avons surtout appris à mieux nous aimer. Je sens aujourd'hui un homme complètement heureux. Évidemment il y a plus de religion dans notre vie. [...] Aujourd'hui je puis jouir pleinement de mon mari et vice-versa. Je suis plus calme, plus paisible et je pèse maintenant 120 livres. [...] J'ai un époux très sincère, assez affectueux, mais qui ne possédait aucune manière de s'exprimer. C'est une chose qui s'apprend puisqu'aujourd'hui, grâce à cette méthode il s'exprime très facilement et tout naturellement, si bien que j'en suis toute bouleversée. Et lorsque vient le temps de la belle rencontre, c'est avec une sincérité qui transperce qu'il me transporte. (lettre 39)

\section{L'impasse morale}

Le problème moral que posait le contrôle des naissances fut ressenti plus cruellement au tournant des années 1960 que par la suite et les lettres qui l'exprimèrent le mieux, curieusement, sont écrites de la main des femmes les plus défavorisées. S'exprimeraient-elles plus ouvertement que les autres? Quoi qu'il en soit, ces femmes font valoir la profondeur du sens religieux dans une couche de la population où on l'attend moins et montrent que le lien entre religion et sexualité était encore bien vivace et douloureusement vécu au tournant des années $1960^{49}$. De nombreuses lettres proviennent de femmes qui se disaient désemparées et avouaient ne pas savoir «comment on peut retarder les naissances sans qu'il y ait péché» (lettre 26). L'une d'entre elles, qui faisait fi des interdits, se plaignait quand même de n'avoir aucune tranquillité d'esprit (lettre 21). Dans une lettre circulaire adressée aux couples qui la consultaient, Rita Breault reconnaissait que le problème de pratiquer la continence périodique et de vivre une vie chrétienne authentique était très grand et que peu de couples en connaissaient la solution ${ }^{50}$.

49. René Hardy, «Les conceptions prénuptiales à Trois-Rivières comme indice de fidélité religieuse, 1850-1945 ", Revue d'histoire de l'Amérique française, 54,4 (printemps 2001) : 531-555. Martine Sevegrand, op. cit., montre que cette tension entre sexualité et religion a été vécue dans les milieux catholiques français des années 1930.

50. Fonds Seréna-Québec, lettre circulaire de Rita Breault en date du 2 décembre 1959. 
Comme les témoignages l'expriment, le problème moral tenait à l'injonction divine $\mathrm{du}$ "Croissez et multipliez-vous » à laquelle les couples se croyaient tenus et à l'impossibilité d'y satisfaire année après année. Il tenait aussi à la contrainte de la continence, qui paraissait plus lourde aux maris qu'aux femmes. Si certains la contestaient, à tous la morale paraissait claire : empêcher la famille par un autre moyen que la continence était un péché mortel.

Des femmes de milieu rural disent avoir consulté leur curé avant de prendre la décision d'arrêter "la manufacture». Probablement cela se faisait aussi à Montréal. Ce n'est qu'à 41 ans, après avoir eu 11 enfants dans des conditions difficiles qu'une femme demanda cette autorisation à son curé (lettre 31). Toujours les motifs invoqués pour un répit ou un arrêt des conceptions apparaissaient des plus valables. Certains confesseurs ne comprenaient pas toujours la difficulté des «carêmes» des couples. À une femme qui eut 5 enfants coup sur coup, puis un sixième, en dépit de bien des privations, son confesseur dit qu'elle n'avait pas jeûné beaucoup, alors qu'elle avait eu une ou deux relations par mois (lettre 26). Elle s'insurgea comme d'autres : «Je ne suis pas mariée à un morceau de bois!» (lettre 22). Le plus difficile était de se voir refuser l'absolution, mais cela est rarement mentionné aussi bien à Montréal qu'ailleurs ${ }^{51}$. Tous les confesseurs n'étaient pas aussi impitoyables et, comme on sait, les noms des bons confesseurs circulaient de bouche à oreille ${ }^{52}$.

Le christianisme croit au péché originel et il professe que, sans la grâce, nous ne sommes pas capables de respecter intégralement et durablement la loi naturelle, dans le domaine sexuel encore moins qu'ailleurs. L'apprentissage de la maîtrise de soi nécessitait le secours de la grâce de Dieu, que l'on puise dans la prière et la pratique des sacrements dont étaient privés les couples onanistes récidivistes ou habitudinaires ${ }^{53}$. Manifestant une déception voilée à l'égard de son Église, dans un entretien qu'il m’a accordé récemment, un prêtre séculier m’expliquait la logique du refus de l'absolution : "Avec le contrôle des naissances vu comme un péché mortel, le prêtre est enfermé dans la logique suivante : s'il n’y a pas

51. Lettre 5 : «Moi je m'étais toujours vue condamner et refuser l'absolution à cause de ce problème»; également lettre 10.

52. Voir D. Baillargeon, op. cit., D. Gauvreau, op. cit., et G. Bouchard, op. cit.. Le phénomène des bons confesseurs est mentionné en entretien par divers informateurs et attesté en France également : Martine Sevegrand, Les enfants du bon Dieu, op. cit., 105, 107.

53. Cardinal Louis-Joseph Suenens, Un problème crucial. Amour et maîtrise de soi (Bruges, Desclée de Brouwer, 1969), 63. 
d'efforts, il n'y a pas de repentir, donc pas de ferme propos et, en conséquence, pas d'absolution ${ }^{54}$.»

Un autre, dans une virulente lettre ouverte adressée aux théologiens en 1966, est allé plus loin dans sa condamnation. Dans la revue Perspectives sociales de l'Université Laval, le père Paul-Eugène Charbonneau, csc, montrait l'impasse dans laquelle le refus de l'absolution jetait les couples en les privant du support nécessaire :

C'est ainsi que le cercle est bouclé, écrivait-il : les époux ne pourraient réussir

à surmonter leurs difficultés que par le recours des sacrements, mais on leur refuse ceux-ci parce qu'ils ne peuvent pas garantir qu'ils surmonteront leurs difficultés. C’est ce qui s'appelle un cercle vicieux parfait. [...] Et le couple repart, condamné à une vie spirituelle marginale, sans espoir et sans Dieu ${ }^{55}$.

Le cercle est bouclé doublement comme le fait état cette lettre déchirante d'une mère de famille nombreuse. Non seulement le couple se retrouvait privé de la grâce nécessaire pour supporter l'épreuve mais, en prime, l'enfant «venait quand même!» :

Après mon mariage, se fut une misère plus terrible. Celle-là tuais la mère, l'épouse, la femme et la raison de vivre. Sans aucun support, car on était en état de péché et le prêtre nous refusais l'absolution pour des grossesses qui venais quand même après s'avoir damné pour les éviter. Avec des pareil idée on priais mais les sacrements était refusé et on se sentais si coupable d'oser s'adresser a Dieu avec de pareil pensée. (lettre 35)

La culpabilité, l'anxiété, la détresse entraînée par la rupture de la communication avec Dieu, autant de sentiments qu'on ne saurait mettre en statistiques, mais qui apparaissent si prégnants dans notre corpus. Et c'est une femme pauvre de tout, sauf de mots, qui fait preuve de cette grande intériorisation de la symbolique religieuse.

Mais il n'y avait pas que le prêtre qui pouvait exclure de la communauté des croyants. Une femme exigeante avec elle-même s'en est d'elle-même exclue. Plutôt que de chercher à extorquer l'absolution à un confesseur compréhensif, elle qui avait donné naissance à 11 enfants, cessa d'ellemême la pratique religieuse tout en gardant contact avec Dieu : «Il y a longtemps que je ne pratiquais plus à cause de ce problème $[\ldots]$ si je ne pratiquais pas à chaque jour je discutais avec lui et savais lui dire merci pour les bons moments.» (lettre 5). Une autre avait le sentiment intime que si elle s'adressait directement à Dieu, Il saurait la comprendre et

54. Prêtre $\mathrm{n}^{\circ}$ 5, entretien d'avril 2001.

55. Perspectives sociales, 21,1 (janvier-février 1966) : 5 . 
même lui offrirait un moyen de satisfaire leur désir d'éloigner la prochaine naissance (lettre 11). Tout cela laisse entendre que l'attitude d'un certain clergé québécois, dans la résolution de ce problème, l'a discrédité aux yeux des fidèles, qui ne le jugeaient plus apte à interpréter la loi de Dieu.

Toutefois, la plupart des couples consultant Seréna cherchaient à se conformer au modèle conjugal prôné par l'Église. En effet, malgré les situations de crise dans lesquelles ils se trouvaient souvent, peu de lettres montrent une révolte ouverte. Quelques-unes pourtant font exception, comme l'illustrent les deux lettres suivantes.

Après avoir égrené le chapelet de ses " 32 ans de misère financière», une femme de ménage montréalaise, mère de 8 enfants et dont le mari est au chômage, fait le récit de son terrible parcours de révolte, heureusement jugulé par la rencontre de Seréna:

Alors tout s'écroule comme un château de cartes. La foi? Oui. Je me dis souvent le soir : mon Dieu aidez-moi. Cela est toute ma prière car ma tête est ailleurs. Je deviens presque folle. [...] Le médecin m’apprend que l'enfant est mort sur moi depuis déjà deux mois et cela est tout la cause de mon mal. Moi je sais que cela est du que j'ai trop travaillé. Alors la je jure que je ne serai plus jamais enceinte. [...]

Alors une personne qui fait la vie rencontrer par hasard m'offre des pilules et je prends le nom. Ma religion? Et bien tant pis! Je n'en veux plus d'enfant. Et la je souffre croyez moi. On me donne un curetage. Avant, puisque je devrai être endormi, on m'envoie le prêtre et je l'envoie promener et je lui dit : si la religion n'a rien trouvé, moi j'ai trouvé des bonnes pilules. Il me confie à un supérieur qui s'occupe de la méthode du thermomètre. Cependant il est prêtre. Ce terrain est délicat pour lui. Il me donne le nom de Mr. et Mad. Gagné [moniteurs] et je n'y crois pas du tout a ce qu'il m'a dit.

D'ailleurs j'espère bien mourir sur cette table ou je va descendre dans quelque minute. Cela il n'y a que moi et ma conscience qui est au courant. Au retour quelle déception, je vie et je prends dix jours à accepter dans le fond de moi la vie que Dieu m’a laissée. La bonté de mon mari fait le reste. [...] La méthode a été sure. De la joi, j’en ai connu. Ma misère a passé et la vie sans toujours avoir peur d'être enceinte. [...] Et j'ai reçu bien souvent l'absolution depuis se temps de grande misère. [...] et il a fallu un dieu qui existe vraiment pour me sauver malgré moi. (Montréal, lettre 10)

Dieu est demeuré toujours présent dans la vie difficile de cette femme : après L’avoir bravé, puis trahi en souhaitant la mort, elle Le bénit. Sa révolte a été jugulée par sa rencontre avec Seréna. Où et comment a-t- 
elle pu trouver le courage de se conformer à cette méthode exigeante, sinon dans la force agissante des représentations religieuses de l'époque?

La seconde lettre vient d'une région éloignée. C’est le premier et le seul cri d'alarme féministe. Elle met en accusation les clivages sociaux, l'incohérence des médecins et leur complaisance vis-à-vis des "grosses madames» riches, fustige un certain clergé nataliste et s'indigne du sort fait aux femmes. Pourtant son auteure cherche une méthode approuvée par l'Église. La force de son sentiment religieux n'est pas ébranlée en dépit de ses difficultés dans le sinistre drame socioreligieux qu'elle dépeint avec ironie :

Mon mari veut de l'appui. Je suis allée voir mon médecin hier. Sa méthode : pendant les menstruations c'est tout. [...] Si vous voulez comprendre mon état, je suis en guerre, contre les pilules que les grosses madames se procurent chez leur spécialiste comme prescriptions s.v.p. et contre les "précautions» que notre médecin nous averti en conscience mais qu'il est prêt à nous vendre. Notre Mgr Curé, lui, voyant le bobo de la paroisse est en chaire et préconise les familles de 12 enfants. Deux extrêmes vous allez me dire. On est en 1964, c'est inconcevable dans le monde de liberté ou nous vivons qu'il n'y ait que la femme qui soit abandonnée. (lettre 37)

Prenant la parole au banquet de la famille terrienne, dans le cadre du Salon de l'agriculture de 1966, le cardinal Léger reconnaissait qu'on pouvait avoir quelques raisons de reprocher à l'Église son intransigeante morale conjugale. Une cristallisation simpliste de son message sous forme de permissions et de défenses a caché sa préoccupation première qui est tout autre, disait-il : l'Église ne cherche qu'à promouvoir l'amour et à assurer sa perfection ${ }^{56}$. Les couples qui ont consulté Seréna et fait un bout de chemin avec eux montraient dans l'ensemble une grande intériorisation de cette philosophie catholique de l'amour nécessaire à Dieu, car Il est l'instrument de son plan créateur :

J'ai lu tout votre livre La limitation des naissances du père de Lestapis que j'ai bien aimer, car il est plus profond et il nous prouve ce que l'homme représente aux yeux de Dieu, à quel point sa virilité est chère à Dieu et on comprend mieux après pourquoi l'Église refuse d'accepter dans ses principes tout ce qui ne se rapporte pas à la continence et il nous prouve aussi combien ce problème est vieux et profond... (Montréal, lettre 7$)^{57}$

56. «Le cardinal Léger précise ce qui préoccupe l'Église : la dignité et la qualité de l’intimité conjugale", Le Devoir, 15 février 1966.

57. Seréna mettait à la disposition de sa clientèle une banque d'ouvrages de morale et de psychologie. Il est fait référence ici au livre du célèbre jésuite français, le père Stanislas de Lestapis, La limitation des naissances (Paris, Édition Spes, 1960). 


\section{CONCLUSION}

Au plus fort de la crise morale que traversaient les milieux catholiques entraînés vers la sécularisation de la société canadienne-française, le groupe Seréna a été un acteur déterminant. La mutation culturelle de la Révolution tranquille n'a pas été aussi subite qu'on pourrait le croire, ainsi que l'écrivait Guy Rocher en 1973, et certains mouvements de l'Action catholique en avaient été les germoirs, selon son expression ${ }^{58}$. Seréna est né de ceux-là.

Il a proposé un système réunissant en un tout morale, philosophie de l'amour et technique contraceptive, suivant la vision du monde catholique. C'était là sa réponse à trois besoins aigus des foyers francophones. Premièrement, à une époque où face au désir et au besoin légitime de limitation des naissances, le sentiment général en était un d'impuissance, Seréna offrait un moyen sûr de régulation des naissances par la continence périodique. Deuxièmement, la méthode qu'il proposait étant admissible par l'Église, Seréna réussit à sortir de l'impasse morale une partie des couples francophones catholiques qui s'efforçaient de vivre chrétiennement. On a vu, dans la correspondance de la clientèle, la détresse morale de ces couples et leur forte intériorisation de la symbolique religieuse. On a vu également leur détresse conjugale et sexuelle et les risques élevés de faillite des mariages. Or, dans les arcanes de l'utilisation de la méthode sympto-thermique, se glissait une éducation à l'amour et au dialogue entre conjoints qui constituait une véritable thérapie conjugale. C’est mon troisième point : Seréna a redonné à ces hommes et à ces femmes le bon usage de leur sexualité, contribuant ainsi à l'établissement de rapports plus égalitaires entre les hommes et les femmes. C'est là réellement où son apport s'est inscrit dans la durée. Mais, si la méthode sympto-thermique a été dépassée par la révolution contraceptive de la pilule et par la baisse spectaculaire de la pratique religieuse, en cette période de transition morale qu'a été le tournant des années 1960, elle a été porteuse d'espoir et salvatrice. Elle a donné un dernier souffle à l'idéal catholique de la maîtrise de soi.

Enfin, en ces temps où tout ce qui touchait la sexualité était honteux et tabou, Seréna, en présentant haut et fort son message dans les médias d'information et dans le vaste auditorium de l'hôpital Notre-Dame, a élargi l'espace de parole et ouvert la voie aux acteurs sociaux qui s'imposeront à la fin des années 1960 : médecins militant en faveur de la contraception et travailleurs sociaux qui prendront le devant de la scène en

58. Guy Rocher, Le Québec en mutation (Montréal, Hurtubise, 1973), 19. 
renouvelant les pratiques et les représentations attachées à la parentalité responsable dans le respect de la liberté de conscience de chacun.

\section{ANNEXE}

Pour la répartition des noms de lieux en catégories, j'ai consulté le Dictionnaire Beauchemin canadien. La catégorie «rural» rassemble les lieux où la municipalité correspond à la paroisse. Le reste est inclus dans "petite ville». Curieusement, il se trouve très peu de lettres de Québec, TroisRivières et Sherbrooke.

\section{Liste des lettres citées}

\begin{tabular}{|c|c|c|c|}
\hline Référence & Date & Destinataire & Provenance \\
\hline 1 & $15-05-1959$ & Mme Rita Breault & Montréal \\
\hline 2 & 22-12-1959 & Mme Rita Breault & Montréal \\
\hline 3 & $12-04-1960$ & Mme Rita Breault & Montréal \\
\hline 4 & 01-02-1961 & M. et Mme Gilles Breault & Montréal \\
\hline 5 & 21-02-1961 & Abbé Jules Parenteau & Montréal \\
\hline 6 & 13-03-1961 & Mme Rita Breault & Montréal \\
\hline 7 & 23-03-1961 & Mme Jeanne Gagné & Montréal \\
\hline 8 & 23-03-1961 & Mme Rita Breault & Montréal \\
\hline 9 & 14-04-1961 & M. et Mme Gagné & Montréal \\
\hline 10 & 01-03-1962 & Mme Jeanne Gagné & Montréal \\
\hline 11 & 08-03-1962 & Mme J. Gagné & Montréal \\
\hline 12 & $12-09-1962$ & Mme Renée Rowan & petite ville \\
\hline 13 & 17-09-1962 & Mme Rita Breault & rural \\
\hline 14 & 04-10-1962 & Mme Rita Breault & rural \\
\hline 15 & 08-10-1962 & Mme Renée Rowan & Montréal \\
\hline 16 & 08-10-1962 & Mme Rita Breault & Montréal \\
\hline 17 & $15-10-1962$ & Monsieur & rural \\
\hline 18 & 27-11-1962 & Mme Rita Breault & Montréal \\
\hline 19 & $12-02-1963$ & Votre enfant Madame & rural \\
\hline 20 & $12-02-1963$ & Votre enfant Madame & rural \\
\hline 21 & $12-02-1963$ & Votre enfant Madame & rural \\
\hline 22 & $12-02-1963$ & Votre enfant Madame & petite ville \\
\hline 23 & $12-02-1963$ & Votre enfant Madame & rural \\
\hline 24 & $12-02-1963$ & Votre enfant Madame & rural \\
\hline 25 & $13-02-1963$ & Votre enfant Madame & rural \\
\hline 26 & $13-02-1963$ & Votre enfant Madame & rural \\
\hline 27 & $13-02-1963$ & Votre enfant Madame & rural \\
\hline 28 & $14-02-1963$ & Votre enfant Madame & rural \\
\hline 29 & $14-02-1963$ & Votre enfant Madame & petite ville \\
\hline 30 & $05-03-1963$ & Mme Rita Breault & Montréal \\
\hline 31 & $17-03-1963$ & Mme Rita Breault & rural \\
\hline 32 & $28-10-1963$ & Mme Rita Breault & Montréal \\
\hline 33 & $25-11-1963$ & Équipe Seréna & petite ville \\
\hline
\end{tabular}


10-02-1964

11-02-1964

20-02-1964

16-04-1964

22-02-1965

22-04-1965

10-11-1965

16-02-1966

28-04-1967

20-12-1967

26-01-1971
Abbé Jules Parenteau

Abbé Jules Parenteau

Équipe Seréna

Équipe Seréna

Mme Rita Breault

Équipe Seréna

Équipe Seréna

Mme Rita Breault

CLES, Seréna

Mme Rita Breault

Mme Rita Breault
Montréal

Montréal

rural

rural

Montréal

Montréal

petite ville

rural

petite ville

petite ville

petite ville 